\title{
Síndrome de obstrucción intestinal distal en pacientes adultos con fibrosis quística. Casos clínicos
}

\author{
CLAUDIO HEINE T. ${ }^{1}$, MARÍA TERESA PARADA C. ${ }^{2}$, \\ RODRIGO GIL D. ${ }^{2}$, FRANCISCO LÓPEZ K. ${ }^{1}$, CARLOS LIZANA S. ${ }^{3}$, \\ MANUEL FERNÁNDEZ A. ${ }^{4}$, RODRIGO QUERA P. ${ }^{5}$
}

Unidad de Coloproctología. Centro de Trasplantes y Enfermedades Respiratorias Departamento de Cirugía. ${ }^{4}$ Unidad de Radiología. Unidad de Gastroenterología. Clínica las Condes. Santiago,

Recibido el 1 de abril de 2009 aceptado el 19 de noviembre de 2009.

Correspondencia a: Dr. Rodrigo Quera P. Unidad de Gastroenterología Lo Fontecilla 441 Las Condes Santiago

Fax: +56-02-6104776 E-mail: rquera@clc.cl

\section{Distal intestinal obstruction syndrome. Report of two cases with cystic fibrosis}

Distal Intestinal Obstruction Syndrome (DIOS) has a 16\% incidence among patients with Cystic Fibrosis (CF). It is characterized by an intestinal obstruction secondary to fecal impaction in distal ileum or cecum. We report two adult patients with DIOS. A female with CF and subjected to a lung transplantation at the age of 13 years. Five years later, she consulted for an intestinal obstruction. She was treated conservatively with a good clinical evolution. She had a new episode of DIOS eight months later, that was also treated conservatively. A 31 year-old male, subjected to bilateral lung transplantation nine years before, that was admitted to the hospital for a bronchiolitis. Three days after admission he started with a intestinal obstruction, that was diagnosed as a DIOS. He was managed conservatively with a good clinical response.

(Rev Med Chile 2010; 138: 68-72).

Key words: Cystic Fibrosis; Intestine Obstruction.
L a fibrosis quística (FQ) es una patología con prevalencia en nuestro país cercana a 1 por cada 4.000 recién nacidos ${ }^{1}$. Estos pacientes presentan una gran variedad de síntomas, siendo los de la esfera gastrointestinal uno de los más frecuentes: $85 \%$ a 90\%. Dentro de ellos, uno de los menos conocidos es el síndrome de obstrucción intestinal distal (SOID) ${ }^{2}$.

En 1941, Rasor y Stevenson describieron por primera vez un cuadro de obstrucción intestinal en un niño con fibrosis quística en el período neonatal ${ }^{3}$ y en la década 1960-69, Jenson utilizó por primera vez el término de equivalente al íleo meconial (meconial ileus equivalent) al encontrar importantes similitudes entre esta enfermedad y la que afecta a recién nacidos con fibrosis quística ${ }^{4}$. En 1981, Park y Grand propusieron el término distal intestinal obstruction syndrome (DIOS) $)^{5}$.

El SOID muestra una incidencia variable entre $8,3 \%$ y $37 \%^{1,2,6-8}$ del total de pacientes portadores de FQ. Si consideramos esta complicación sólo en adultos y adolescentes con FQ alcanza una incidencia de $16 \%^{7,9,10}$. En el caso de pacientes sometidos a trasplante pulmonar por fibrosis quística, la incidencia es mayor, alcanzando $20 \%{ }^{11}$. La mayoría de los pacientes presentan su primer episodio después de los 18 años y cerca de la mitad de ellos tienen más de un episodio durante su vida ${ }^{12}$. Hasta la fecha no existen casos publicados en nuestro país de pacientes adultos con FQ sometidos a trasplante pulmonar y que hayan presentado esta complicación.

\section{Caso Clínico 1}

Paciente de sexo femenino con diagnóstico de fibrosis quística desde el nacimiento y sometida a un trasplante pulmonar bilateral secuencial a los 13 años (2003). En octubre de 2008 consultó por 
Síndrome de obstrucción intestinal distal en pacientes adultos con fibrosis quística - C. Heine T.

cuadro de 4 días de dolor abdominal en ambos hipocondrios con ausencia de deposiciones y gases por ano. En el examen físico de ingreso sólo destacó un abdomen distendido sin signos peritoneales. Los exámenes de laboratorio mostraron anemia leve (hematocrito 32,7\%, hemoglobina: $11 \mathrm{~g} / \mathrm{dl}$ ) y una PCR discretamente elevada (1 mg/Lt). Se solicitó una radiografía simple de abdomen que mostró dilatación hidroaérea de asas de intestino delgado proximal y el resto de las asas intestinales con abundante contenido fecal (Figura 1a). En este escenario se decide administrar profilaxis antibiótica (ceftriaxona/metronidazol) e iniciar solución PEG 3.350 vía oral asociado a proctoclisis ( $1.000 \mathrm{cc}$ de agua destilada $+15 \mathrm{~g}$ de bicarbonato de sodio $+10 \mathrm{ml}$ de vaselina líquida a pasar en 2 horas por vía rectal) evolucionando con vómitos biliosos y aumento del dolor abdominal en fosa iliaca derecha sin presentar deposiciones. Se so-

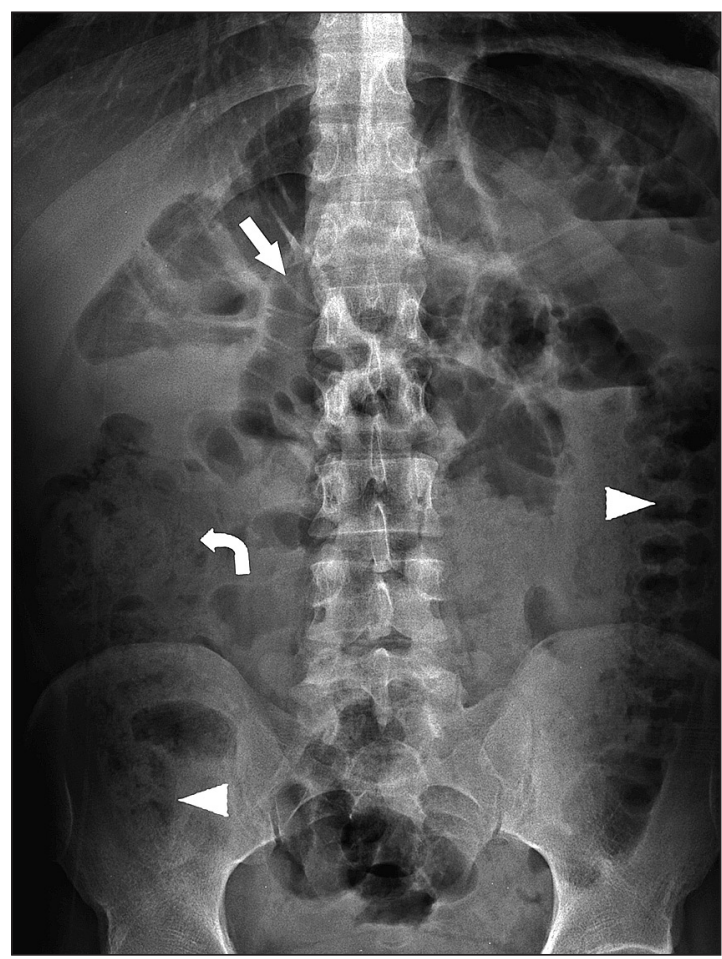

Figura 1a. Radiografía de abdomen simple (decúbito). El yeyuno aparece distendido y con aire (flecha recta), el íleon se muestra con contenido de aspecto estercoráceo (flecha en ángulo); el colon aparece relativamente colapsado en fosa ilíaca derecha y flanco izquierdo (cabeza de flecha). licitó una tomografía computada de abdomen y pelvis que mostró la presencia de contenido estercoráceo desde 40 a $50 \mathrm{~cm}$ de íleon distal hasta el ciego y colon transverso proximal confirmándose el diagnóstico de SOID (Figura 1b). Dada la poca tolerancia y poca respuesta al uso de PEG por vía oral y proctoclisis, se administró medio de contraste por sonda nasogástrica (Gastrografin ${ }^{\circledR} 100 \mathrm{ml}$ diluidos en $400 \mathrm{ml}$ de solución fisiológica) con lo que evacúa gran cantidad de deposiciones líquidas y una importante mejoría de su cuadro clínico. La radiografía simple de abdomen de control mostró desaparición de las asas de intestino delgado dilatadas en el abdomen superior. Fue dada de alta al cuarto día quedando con aporte de enzimas pancreáticas. A pesar de este tratamiento, la paciente presentó un nuevo cuadro de SOID 8 meses después del alta. Este nuevo episodio fue tratado desde un comienzo con Gastrografin ${ }^{\circledR}$ presentan-

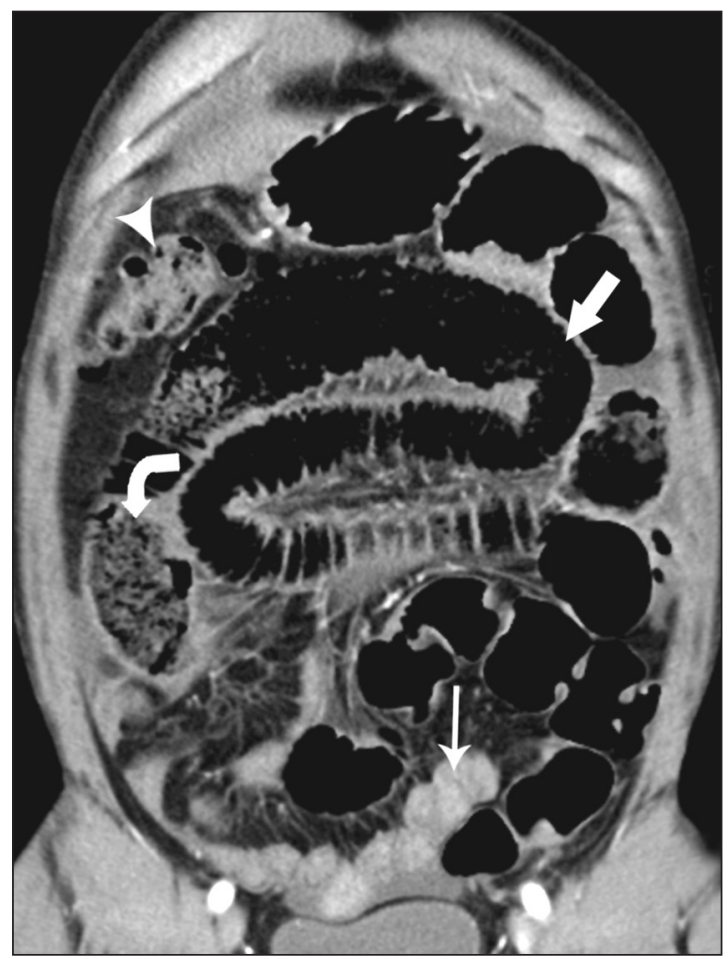

Figura 1b. Tomografía computada, visión coronal y sin contraste oral. El yeyuno se encuentra muy dilatado y con aire (flecha recta); el íleon proximal presenta contenido de aspecto estercoráceo (flecha en ángulo) y el íleon distal está colapsado con contenido de mayor densidad de lo habitual (flecha recta fina); el colon aparece parcialmente colapsado (cabeza de flecha). 
do muy buena respuesta. En la actualidad, y tras 10 meses del primer cuadro, no ha presentado nuevos episodios de SOID como tampoco dolor abdominal ni constipación.

\section{Caso Clínico 2}

Paciente de 31 años con antecedentes de FQ diagnosticada a los 2 años de edad con tratamiento y controles regulares hasta mayo de 2000, año en que se le realizó un trasplante pulmonar bilateral, con muy buena evolución hasta 2009 . El cuadro actual se caracterizó por una descompensación respiratoria diagnosticada como una bronquiolitis obliterante siendo hospitalizado para su tratamiento. Al tercer día de hospitalización refirió un dolor abdominal tipo cólico de intensidad progresiva, localizado mayormente en hemiabdomen inferior derecho, asociado a 3 episodios de vómitos alimentarios. En el examen

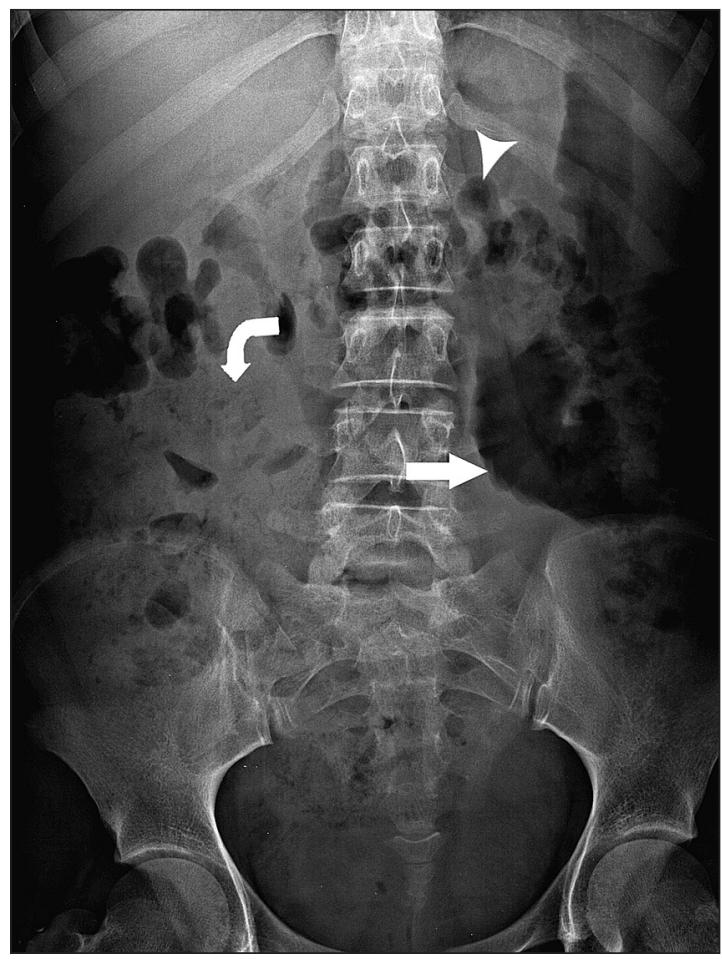

Figura 2a. Radiografía de abdomen simple. El íleon se encuentra distendido y con aire (flecha recta) y presenta contenido en su interior (flecha en ángulo); el colon se muestra relativamente colapsado (cabeza de flecha). físico el abdomen se palpaba distendido y sensible en hemiabdomen derecho con bazuqueo. Se decidió tomar una radiografía simple de abdomen que mostró una moderada dilatación de asas de intestino delgado en hemiabdomen superior además de la presencia de abundante aire y contenido estercoráceo en todo el marco cólico (Figura 2a). Se solicitó además una tomografía computada de abdomen que mostró una importante dilatación de intestino delgado en la porción más alta del yeyuno hasta la parte proximal del íleon que parecían ocupados y distendidos por material de aspecto fecaloideo (Figura 2b), concluyendo con el diagnóstico de SOID. Se inició tratamiento con una dosis de Gastrografin ${ }^{\circledR}$ (100 ml diluidos en $400 \mathrm{ml}$. de suero fisiológico) presentando al cabo de 2 horas, gran cantidad de deposiciones líquidas e importante disminución de los síntomas. La radiografía simple de abdomen de control mostró distensión de algunas asas de intestino delgado en menor grado que en el examen anterior además de

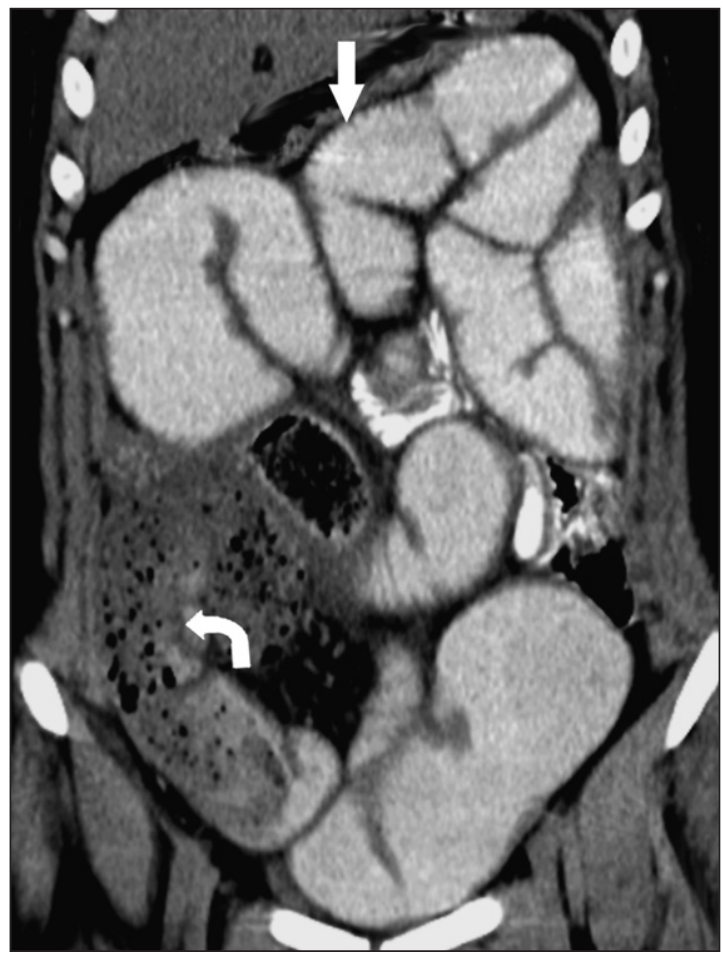

Figura 2b. Tomografía computada, visión coronal. El yeyuno, con contraste oral aparece distendido (flecha recta); el íleon presenta contenido en su interior de aspecto estercoráceo (flecha en ángulo). 
la presencia de medio de contraste en el intestino distal y marco colónico. El paciente fue dado de alta con suplemento de enzimas pancreáticas, encontrándose en buenas condiciones tras 10 meses de su hospitalización sin presentar nuevos cuadros de dolor abdominal, distensión o constipación.

\section{Discusión}

El SOID es una patología única en los pacientes con FQ caracterizada como una obstrucción intestinal asociada a la acumulación de material fecal altamente viscoso en el íleon terminal y en el hemicolon derecho, como el resultado de una combinación de insuficiencia pancreática exocrina, acumulación de moléculas intraluminales, mucosidad intestinal anormal y un tránsito intestinal más lento a través del intestino delgado ${ }^{10,13}$. Existen factores que pueden precipitar el SOID, como infecciones respiratorias intercurrentes, deshidratación, inmovilizaciones prolongadas o una pobre ingesta dietética ${ }^{14}$.

Los síntomas más frecuentes son dolor abdominal recurrente con distensión, constipación, anorexia, saciedad precoz y vómitos. En el examen físico, muchas veces es posible encontrar una masa abdominal en fosa ilíaca derecha ${ }^{2,12,13,15}$, la que muchas veces es dolorosa a la palpación. El diagnóstico diferencial en pacientes con FQ debe incluir la invaginación intestinal, la obstrucción por adherencias, apendicitis aguda o una enfermedad de Crohn ${ }^{10,16}$. Usualmente el diagnóstico se realiza con los antecedentes de FQ, una historia detallada del cuadro clínico, examen físico acucioso, una radiografía de simple abdomen y una tomografía computada de abdomen ${ }^{10}$.

Existe gran variedad de alternativas terapéuticas no invasivas descritas en la literatura, siendo algunas de ellas: la instalación de enemas fosfatados asociados a laxantes orales (aceite mineral o sulfato de magnesio), una baja dosis de solución de electrolitos, N-Acetilcisteína, neostigmina endovenosa, la administración de una solución de polietilenglicol asociado a electrolitos (PEG$\left.3350^{\circledR}\right)$ o el uso de enzimas pancreáticas ${ }^{8,13,16,17,18}$. Sin embargo, algunos de estos medicamentos presentan inconvenientes al no recomendarse su uso en niños menores de 12 años o porque el uso continuo de enzimas pancreáticas provocaría un daño crónico de la mucosa colónica, llegando incluso hasta la colonopatía fibrosante ${ }^{16}$.
En el caso del medio de contraste hiperosmolar (Gastrografin ${ }^{\circledR}$ ), ya en 1969 apareció el primer trabajo describiendo su uso en enema rectal para el tratamiento del íleo meconial en pacientes con fibrosis quística ${ }^{19}$. Más tarde, Burke y cols demostraron que el uso de Gastrografin ${ }^{\circledR}$ y el surfactante son los agentes más efectivos en aliviar el cuadro de constipación sin provocar daños en la mucosa intestinal al ser probados por vía rectal en ratas $^{20}$.

Gastrografin $^{\circledR}$ (Schering, Berlín, Alemania), es un medio de contraste soluble en agua y largamente utilizado tanto desde un punto de vista diagnóstico como terapéutico. La composición de $100 \mathrm{ml}$ de Gastrografin ${ }^{\circledR}$ contiene $10 \mathrm{~g}$ de amidotrizoato sódico, $66 \mathrm{~g}$ de amidotrizoato de meglumina y $37 \mathrm{~g}$ de yodo asociados a un agente humectante (polisorbato 80). Su osmolaridad alcanza los $1.900 \mathrm{mosm} / \mathrm{Lt}$ lo que es casi 6 veces la osmolaridad del líquido extracelular, permitiendo el movimiento de agua dentro del lumen intestinal, logrando una disminución de la viscosidad del contenido intraluminal, disminución del edema de la pared del intestino delgado y un aumento de la contractilidad del músculo liso lo que facilita la evacuación del material fecal y la resolución del cuadro obstructivo. Su vida media es de 30 a 60 minutos, y no presenta absorción intestinal ${ }^{8,11,21}$. La vía de administración puede ser por vía oral, a través de una sonda nasogástrica, por vía rectal o su uso mediante instilación durante la colonoscopia de un caso agudo de SOID ${ }^{14}$. O’Halloran y cols demostraron una efectividad de $81 \%$ con sólo una dosis, siendo $75 \%$ de estos pacientes tratados en forma ambulatoria ${ }^{8}$. Los principales efectos secundarios del Gastrografin ${ }^{\circledR}$ son diarrea, náuseas y vómitos ${ }^{20,21}$ por lo que no debiera ser indicado en pacientes con deshidratación intensa, obstrucción prologada, evidencia de abdomen agudo quirúrgico o en casos de sensibilidad conocida al yodo ${ }^{8,22}$.

Durante el episodio agudo, la cirugía presenta una alto riesgo de morbimortalidad debido a sangrado, cicatrización deficiente y a infecciones pulmonares postoperatorias en un paciente con inmunosupresión asociada ${ }^{8,22}$, por lo que debiera reservarse exclusivamente para los casos de fracaso al tratamiento médico o en presencia de complicaciones como obstrucción intestinal completa por adherencias asociadas, invaginación, vólvulo o perforación intestinal ${ }^{2,10,18}$.

En resumen, el SOID es una complicación que debe ser considerada principalmente en pacientes 
adultos con FQ y sobre todo en aquellos que han recibido un trasplante pulmonar. En esta patología, la alta sospecha clínica sumada al apoyo de un equipo multidisciplinario (gastroenterólogo, neumólogo, cirujano, radiólogo) permite evitar una cirugía en estos pacientes. En cuanto al tratamiento, el uso de Gastrografin ${ }^{\circledR}$ demuestra ser una estrategia segura, efectiva y de bajo costo en estos pacientes.

\section{Referencias}

1. Sánchez I, Pérez MA, Boza ML, Lezana V, Vila MI, Repetto $\mathrm{G}$, et al. Consenso nacional de fibrosis quística. Rev Chil Pediatr 2001; 72: 356-80.

2. Hanly J, Fitzgerald M. Meconium Ileus equivalent in older patients with cystic fibrosis. BMJ 1983; 286: 141113.

3. Rasor R, Stevenson C. Cystic fibrosis of the pancreas: a case history. Rocky Mountain Med J 1941; 38: 218-20.

4. Jenson K. Meconium ileus equivalent in a 15 -year-old patient with mucoviscidosis. Acta Paediatr 1962; 51: 344-48.

5. Park R, Grand R. Gastrointestinal manifestations in cystic fibrosis. Gastroenterology 1981; 81: 1143-61.

6. Koshoo V, Udall J. Meconium ileus equivalent in children and adults. Am J Gastroenterol 1994; 89; 153-57.

7. Dray X, Bienvenu T, Desmazes-Dufeu N, Dusser D, Marteau P, Hubert D. Distal intestinal obstruction syndrome in adults with cystic fibrosis. Clin Gastroenterol Hepatol 2004; 2: 498-503.

8. O'Halloran SM, Gilbert J, Mckendrick OM, Carty H, Heaf DP. Gastrografin in acute meconium ileus equivalent. Arch Dis Child 1986; 61: 1128-30.

9. Penkhet A, Wise A, Mearns M, Hodson M, Batten J. Cystic fibrosis in adolescents and adults. Thorax 1987; 42: 526-32.

10. Nassenstein K, Schweiger B, Kamler M, Stattaus J, Lauenstein T, Barkhausen J. Distal intestinal obstruction syndrome in the early postoperative period after lung transplantation in a patient with cystic fibrosis: morphological findings on computed tomography. Gut 2005; 54: 1662-63.

11. Gilljam M, Chaparro C, Tullis E, Chan $\mathrm{CH}$, Keshavjee $\mathrm{SH}$, Hutcheon M. GI complications after lung transplantation in patients with cystic fibrosis. Chest 2003; 123: 37-41.

12. Shah U, Shafiq Y, Khan M. Gastrograffin use in distal intestinal obstruction syndrome of cystic fibrosis. J Ayub Med Coll Abbottabad 2007; 19: 58-60.

13. Kurtzman T, Borowitz S. Successful use of neostigmine in a patient with refractory distal intestinal obstruction syndrome. J Pediatr Gastroenterol Nutr 2002; 35: 700-3.

14. Shidrawi R, Murugan N, Westaby D, Gyi K, Hodson M. Emergency colonoscopy for intestinal distal obstruction syndrome in cystic fibrosis patients. Gut 2002; 51: 285-86.

15. Agrons G, Corse W, Markowitz R, Suárez E, Perry D. Gastrointestinal manifestations of cystic fibrosis: Radiologic-pathologic correlation. Radiographics 1996; 16: 871-90.

16. Jackson R, Pencharz P. Cystic Fibrosis. Best Pract Res Clin Gastroenterol 2003; 17: 213-35.

17. Koletzko S, Stringer D, Durie P. Lavage treatment of distal intestinal obstruction syndrome in children with cystic fibrosis. Pediatrics 1989; 83: 727-33.

18. Andersen H, Hjelt K, Waever E, Overgaard K. The agerelated incidence of meconium ileus equivalent in a cystic fibrosis population: the impact of high energy intake. J Ped Gastro Nutr 1990; 11: 356-60.

19. Noblett $H$. Treatment of uncomplicated meconium ileus by gastrograffin enema: A preliminary report. J Pediatr Surg 1969; 4: 190-7.

20. Burke M, Ragi J, Karamanoukian H, Kotter M, Brisseau G, Borowitz D, et al. New strategies in nonoperative management of meconium ileus. J Pediatr Surg 2002; 37; 760-64.

21. Abbas SM, Bissett IP, Parry BR. Meta-analysis of oral water soluble contrast agent in the management of adhesive small bowel obstruction. Br J Surg 2007; 94: 404-11.

22. Escobar M, Grosfeld J, Burdick J, Powell R, Jay C, Wair A, et al. Surgical considerations in cystic fibrosis: A 32-year evaluation of outcomes. Surgery 2005; 138: 560-72. 